\title{
Acute Erythroid Leukemia in Remission
}

National Cancer Institute

\section{Source}

National Cancer Institute. Acute Erythroid Leukemia in Remission. NCI Thesaurus. Code C7466.

Acute erythroid leukemia which is not growing and responds to treatment. 